\title{
Biochemical Characterization of a SET and Transposase Fusion Protein, Metnase: Its DNA Binding and DNA Cleavage Activity ${ }^{\dagger}$
}

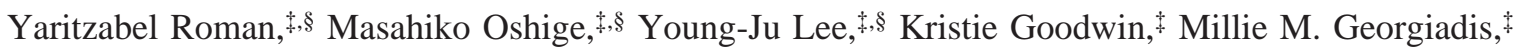 \\ Robert A. Hromas," and Suk-Hee Lee*,\$,\$ \\ Department of Biochemistry and Molecular Biology and Walther Cancer Institute, Indiana University School of Medicine, \\ Indianapolis, Indiana 46202, and Department of Internal Medicine and Cancer Treatment and Research Center, University of \\ New Mexico, Albuquerque, New Mexico 87131
}

Received March 20, 2007; Revised Manuscript Received July 27, 2007

\begin{abstract}
Metnase (SETMAR) is a SET and transposase fusion protein that promotes in vivo end joining activity and mediates genomic integration of foreign DNA. Recent studies showed that Metnase retained most of the transposase activities, including 5'-terminal inverted repeat (TIR)-specific binding and assembly of a paired end complex, and cleavage of the $5^{\prime}$-end of the TIR element. Here we show that R432 within the helix -turn-helix motif is critical for sequence-specific recognition, as the R432A mutation abolishes its TIR-specific DNA binding activity. Metnase possesses a unique DNA nicking and/or endonuclease activity that mediates cleavage of duplex DNA in the absence of the TIR sequence. While the HTH motif is essential for the Metnase-TIR interaction, it is not required for its DNA cleavage activity. The DDElike motif is crucial for its DNA cleavage action as a point mutation at this motif (D483A) abolished its DNA cleavage activity. Together, our results suggest that Metnase's DNA cleavage activity, unlike those of other eukaryotic transposases, is not coupled to its sequence-specific DNA binding.
\end{abstract}

Metnase (SETMAR) contains two functional domains: a $\mathrm{SET}^{1}[\mathrm{Su}($ var)3-9, Enhancer-of-zeste, Trithorax $]$ domain and a transposase domain $(1-4)$. The Metnase SET domain is associated with histone lysine methyltransferase activity at histone 3, lysine 4, and lysine 36 (2), while the transposase domain, containing the acidic DDE motif, a conserved motif among retroviral integrase and transposase families, is essential for strand transfer and end joining activities $(5,6)$. We recently identified a novel double-strand break (DSB) repair factor, Metnase, that promotes in vivo end joining activity and mediates genomic integration of foreign DNA (2). Both activities are likely required for Metnase's role in DSB repair and genomic integration since deletion of either SET or transposase domain abolished its function in vivo (2).

† This research was supported by grants from the NIH (CA92111 to S._H.L. and CA102283 and HL07578 to R.A.H.) and the U.S. Army (DAMD17-00-1-0295 to S.-H.L.).

* To whom correspondence should be addressed: IU Cancer Research Institute (Rm 153), 1044 W. Walnut St., Indianapolis, IN 46202. Phone: (317) 278-3464. Fax: (317) 274-8046. E-mail: slee@ iupui.edu.

$\doteqdot$ Department of Biochemistry and Molecular Biology, Indiana University School of Medicine.

$\S$ Walther Cancer Institute, Indiana University School of Medicine.

"University of New Mexico.

${ }^{1}$ Abbreviations: DSB, double-strand break; DTT, dithiothreitol; DMEM, Dulbecco's modified Eagle's medium; FANCD2, Fanconi Anemia Complementation Group D2 protein; HLMT, histone lysine methyltransferase; HTH, helix - turn-helix; MBS, Metnase binding site(s); NBS1, Nijmegen breakage syndrome; PAGE, polyacrylamide gel electrophoresis; pBS, pBluescript; PEC, paired end complex; RFI, supercoiled replicative form I DNA; RFII, nicked relaxed DNA; RFIII, linearized duplex DNA; SET, Su(var)3-9, Enhancer-of-zeste, Trithorax; TIR, 5'-terminal inverted repeat.
Although approximately half of the human genome derives from transposable elements (7), very little is known about transposase-like functions in higher eukaryotes. RAG1/2 recombinase can mediate transposition of DNA flanked by a specific recombination signal sequence, and the mechanism of V(D)J recombination by RAG1/2 shares similarities with those of the bacterial and Mariner transposons (8). A plant transposase, DAYSLEEPER, was found to be essential for proper growth, suggesting that a transposase may have a normal physiologic cellular function outside of transposition of genetic elements, although the mechanism of its action was not clear $(9,10)$. Metnase (SETMAR) possesses most of the transposase's functions, including sequence-specific DNA binding and DNA looping $(1,11)$, assembly of a paired end complex (PEC), cleavage of the $5^{\prime}$-end of the TIR element, and integration at a TA dinucleotide target site (11). Given that DNA transposons are generally accepted as being extinct in humans (7), these activities may have a role in other DNA metabolism such as DNA repair (2). In this study, we found that the helix-turn-helix (HTH) motif within the transposase domain is essential for the sequence-specific DNA binding, but not for DNA cleavage of Metnase. It possesses unique DNA cleavage activity that mediates cleavage of duplex DNA in a TIR-independent manner. We discuss a potential role for Metnase (SETMAR) DNA binding and DNA cleavage activities in DNA repair and gene regulation.

\section{MATERIALS AND METHODS}

Chemicals, DNA Substrates, and Antibodies. The following suppliers provided the listed items: [ $\left.\gamma{ }^{32} \mathrm{P}\right] \mathrm{ATP}(3000 \mathrm{Ci} /$ mmol) from Perkin-Elmer and Analytical Science (Boston, 
MA), DE81 filters from Whatman Bio System (Maidstone, England), heparin-Sepharose from Amersham Biosciences (Piscataway, NJ), and Bradford reagents and protein molecular weight markers from Bio-Rad (Hercules, CA). An antiMetnase antiserum (polyclonal) was generated from rabbits using two peptides representing amino acids 483-495 (DEKWILYDNRRRS) and 659-671 (WQKCVDCNGSYFD). An anti-FLAG antibody was obtained from Sigma (St. Louis, MO). The oligonucleotides and the $5^{\prime}$-fluorescently labeled DNA were obtained from Integrated DNA Technologies (Coralville, IA).

Cell Cultures and Preparation of Cell Extracts. Human 293 cells overexpressing FLAG-Metnase were grown in Dulbecco's modified Eagle's medium (DMEM) (GibcoBRL) supplemented with $10 \%$ fetal bovine serum (GibcoBRL) and antibiotic-antimycotic reagent (10 units $/ \mathrm{mL}$ penicillin $\mathrm{G}, 10 \mu \mathrm{g} / \mathrm{mL}$ streptomycin, and $0.25 \mu \mathrm{g} / \mathrm{mL}$ amphotericin B) (Gibco-BRL). Cell extracts were prepared by scraping culture dishes $(150 \mathrm{~mm})$ after they had been washed with PBS. Cells in the exponentially growing phase were used for the preparation of cell extracts.

Expression and Purification of FLAG-Metnase. The cDNA for human Metnase (SETMAR) was subcloned into pFLAGCMV-2 (Sigma) at HindIII and BamHI sites. For PCR amplification, the forward primer (5'-CCC AAG CTT GGG ATG GCG GAG TTT AAG GAG-3') and the reverse primer (5'-CGC GGA TCC GCG TTA ATC AAA ATA GGA ACC ATT ACA-3') were used. Positive clones were identified by restriction analysis and DNA sequencing and were transfected into human 293 cells for protein expression. Metnaseexpressing cells $\left(1.0 \times 10^{6}\right)$ were suspended in $1 \mathrm{~mL}$ of extraction buffer (TEGDN) [50 mM Tris-HCl (pH 7.5), 1 mM EDTA, $10 \%$ glycerol, 5 mM DTT, 1.0\% Nonidet-P40, and mammalian protease inhibitor cocktails containing 0.2 $\mathrm{M} \mathrm{NaCl}$ ] and centrifuged $(100000 \mathrm{~g}$ ) for $30 \mathrm{~min}$ following a brief vortex. The supernatant (S100 fraction) was filtered through Whatman paper and used for protein purification. The S100 fraction was incubated at $4{ }^{\circ} \mathrm{C}$ for $60 \mathrm{~min}$ with anti-FLAG M2 affinity gel (Sigma) that had been preequilibrated with TEGDN buffer containing $0.2 \mathrm{M} \mathrm{NaCl}$. The beads were washed three times with TEGDN and 2.0 $\mathrm{M} \mathrm{NaCl}$ prior to elution of the protein with TEGDN and 0.2 $\mathrm{M} \mathrm{NaCl}$ containing FLAG peptide $(500 \mu \mathrm{g} / \mathrm{mL})$. The eluant was diluted with 10 volumes of TEGDN buffer and loaded onto a heparin-Sepharose 6 Fast Flow column (Amersham Biosciences) pre-equilibrated with TEGDN buffer. After the column had been washed, Metnase (SETMAR) protein was fractionated using a linear gradient (from 0 to $2.0 \mathrm{M} \mathrm{NaCl}$ ) of TEGDN buffer. The eluted protein was then dialyzed against TEGDN buffer containing $50 \mathrm{mM} \mathrm{NaCl}$.

SDS-PAGE and Western Blot Analysis. Protein fractions were analyzed by $10 \%$ SDS-polyacrylamide gel electrophoresis (SDS-PAGE). For visualization, the gel was silverstained using RAPID-AG-STAIN (ICN Biomedical, Inc.). Protein was then transferred to a polyvinylidene difluoride (PVDF) membrane and probed with an anti-FLAG (monoclonal mouse IgG, Sigma) or an anti-Metnase antibody (polyclonal rabbit $\mathrm{IgG}$ ) followed by horseradish peroxidaseconjugated secondary antibody. Protein was visualized by using the ECL system (Amersham Biosciences).

DNA Cleavage Assay. For the RFI cleavage assay, the reaction mixtures contained $25 \mathrm{mM}$ Tris- $\mathrm{HCl}(\mathrm{pH} 7.5), 100$ ng of supercoiled pBluescript II SK+ phagemid DNA (pBS), the indicated amount of either $\mathrm{MgCl}_{2}$ or $\mathrm{MnCl}_{2}, 10 \mathrm{mM}$ DTT, and the indicated amount of Metnase. The mixtures were incubated at $37{ }^{\circ} \mathrm{C}$ for $60 \mathrm{~min}$. After incubation, the mixtures were subjected to $1 \%$ agarose gel electrophoresis in TBE (Tris-boric acid-EDTA) buffer following addition of $5 \mu \mathrm{L}$ of sample loading buffer consisting of 5\% sarkosyl, $0.0025 \%$ bromophenol blue, and 25\% glycerol. DNAs on agarose gel were stained with ethidium bromide, visualized on a UV transilluminator, and analyzed with the NIH image system (version 1.62). For the DNA cleavage assay using oligonucleotides, reaction mixtures $(20 \mu \mathrm{L})$ containing $\left[5^{\prime}-{ }^{32} \mathrm{P}\right]-$ dsDNA (200 fmol) were incubated with indicated amounts of Metnase in the presence of $1 \mathrm{mM} \mathrm{MgCl} 2$. After incubation at $37{ }^{\circ} \mathrm{C}$ for $60 \mathrm{~min}$, reaction mixtures were analyzed on a $16 \%$ denaturing polyacrylamide gel for DNA cleavage.

Analysis of DNA Cleavage Sites. To examine the cleavage sites, linearized pBS (RFIII) DNA was isolated from agarose gel following incubation of RFI DNA with Metnase. Following end labeling of gel-isolated RFIII DNA with $\left[\gamma_{-}{ }^{32} \mathrm{P}\right]-$ ATP and T4 phosphonucleotide kinase (Amersham Biosciences), DNA was purified using the CENTRI SPIN-20 gel filtration kit. Approximately $20 \mathrm{fmol}$ of ${ }^{32} \mathrm{P}$-labeled RFIII DNA $(10000 \mathrm{cpm})$ was incubated with either EcoRI (10 units) or Scal (10 units) at $37{ }^{\circ} \mathrm{C}$ for $60 \mathrm{~min}$. Following incubation, DNA samples were analyzed by $2.0 \%$ agarose gel electrophoresis, the gel from which was then dried on DE81 paper (Whatman) and exposed to BioMax MS film (Kodak, Rochester, NY). The RFIII products were treated with EcoRI, and the DNA fragments were cloned into the EcoRI and EcoRV sites of pBS. Following transformation of ligated DNA into Escherichia coli DH5 $\alpha$, plasmid DNAs containing an insert with fewer than 200 nucleotides were selected and analyzed for the cleavage site by DNA sequencing. The nucleotide sequence of each clone was determined with a Perkin-Elmer/Applied Biosystems 3100 Genetic Analyzer and Big Dye Terminator chemistry system and analyzed with DNASIS-Mac, version 3.2 (Hitachi Software Engineering Co.), and GENETYX-MAC, version 9.0 (Software Development Co., Ltd.).

Expression and Purification of dS-Metnase(329-671) and dS-Metnase (433-671). On the basis of sequence comparisons with Hsmar1, an ancient mariner transposon found in the human genome, the transposase domain of Metnase (residues 329-671) was cloned by PCR of the full-length Metnase cDNA. PCR oligonucleotides were designed for the purpose of creating restriction sites for cloning: $5^{\prime}$-ACCCTTGAGCATATGAAAATGATGTTAGACAAA-3' (NdeI on the $5^{\prime}$-end) and 5'-ATCCTCGAGTTAATCAAAATAGGAACCATT-3' (XhoI on the $3^{\prime}$-end). The fragment was cloned into pET15b using NdeI and XhoI sites, and the ligated plasmid was used to express a six-His-tagged protein in Rosetta. The protein was initially purified by Ni-NTA affinity chromatography followed by S-Sepharose ion exchange chromatography. The six-His tag was removed by thrombin digestion, and the protein was then further purified by S-Sepharose ion exchange chromatography. Typical yields were $3-5 \mathrm{mg} / \mathrm{L}$ of culture. To probe the domain structure, we conducted limited proteolysis of the 329-671 protein using trypsin (Figure 8). This experiment resulted in several products, including an $\sim 25 \mathrm{kDa}$ band that was sent for $\mathrm{N}$-terminal sequencing analysis and found to have an 
A.

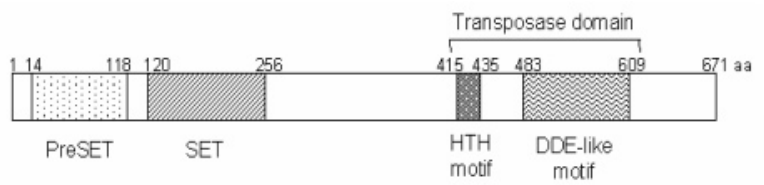

B.
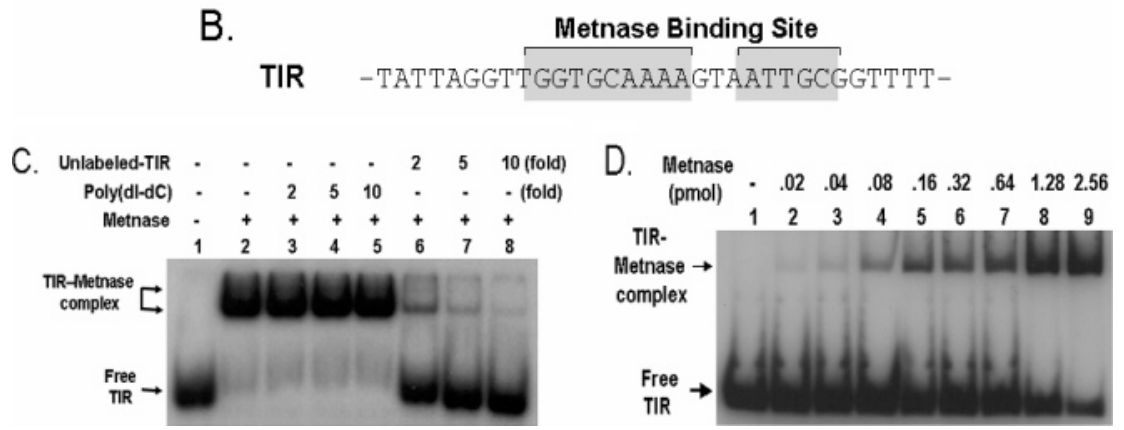

FIgURE 1: (A) Schematic diagram of human Metnase (SETMAR). The PreSET domain contains a cysteine- and histidine-rich putative $\mathrm{Zn}^{2+}$ binding motif. The SET domain has the histone lysine methyltransferase motif. The transposase domain contains the conserved HTH and DDE-like motifs. (B) DNA sequence of the 5'-terminal inverted repeats (TIR). The two Metnase binding sites are colored gray. (C) Gel mobility shift analysis of the Metnase-TIR complex. The in vitro DNA binding activity of Metnase with [5'-32P]TIR DNA was examined in the presence of increasing amounts of either unlabeled poly(dI-dC) or TIR. See Materials and Methods for details. (D) Gel mobility shift analysis of the Metnase-TIR interaction. The indicated amount of Metnase was added to 5'-32P-labeled TIR (200 fmol) for the MetnaseTIR interaction on a gel mobility shift assay.

$\mathrm{N}$-terminus beginning with residue 433 . The molecular mass of this fragment, based on SDS-PAGE analysis, was consistent with removal of the just the N-terminal residues as the predicted size for the $433-671$ protein is $\sim 28 \mathrm{kDa}$. This protein fragment including residues 433-671 from Metnase was cloned by PCR using 5'-TCTACGGTCCATATGCATTTGAAGCAAATTGAA- $3^{\prime}$ with the previous $3^{\prime}$-oligonucleotide, and the protein was purified as described above.

Electrophoretic Mobility Shift Assay of the Metnase-DNA Interaction. Duplex DNA was labeled with $\left[\gamma_{-}{ }^{32} \mathrm{P}\right] \mathrm{ATP}$ (ICN) and T4 polynucleotide kinase (Roche Molecular Biochemical) on the basis of manufacturer's instructions. The indicated amount of wt-Metnase or the mutant was incubated with 200 fmol of $5^{\prime}{ }^{32} \mathrm{P}$-labeled DNA at room temperature for $15 \mathrm{~min}$ in a reaction mixture containing $50 \mathrm{mM}$ Hepes- $\mathrm{KOH}(\mathrm{pH}$ 7.8), poly(dI:dC) $(0.2 \mu \mathrm{g})$, bovine serum albumin $(0.2 \mu \mathrm{g} /$ $\mu \mathrm{L}$ ), and $50 \mathrm{mM} \mathrm{NaCl}$. The Metnase-DNA complex was analyzed on $5 \%$ polyacrylamide gels in $0.5 \times$ TBE (43.2: 0.8 acrylamide:bisacrylamide ratio). The gels were dried and exposed to X-ray film (Kodak). For quantification, the bands of interest were excised from the gels and measured for radioactivity using a Beckman LS 6500 scintillation counter.

\section{RESULTS}

Interaction of Metnase with TIR Sequence. Metnase (SETMAR) is a novel DNA repair protein consisting of a SET domain with histone lysine methyltransferase (HLMT) activity and a transposase domain (Figure 1A (1-4). It possesses a sequence-specific DNA binding activity that recognizes the 19-mer core of the 5 '-terminal inverted repeats (TIRs) of the Hsmar1 element (Figure 1B) (1). Given that the human chromosomes contain more than 7000 potential Metnase (SETMAR) binding sites (1), Metnase with its histone methylation activity may form a unique gene regulatory network in the human genomes $(4,11)$. In this study, we biochemically characterized the Metnase-TIR interaction. Metnase was purified from human 293 cells transfected with a mammalian vector expressing FLAGMetnase. Purified Metnase formed a stable interaction with $\left[5^{\prime}-{ }^{32} \mathrm{P}\right] \mathrm{TIR}$ that was significantly inhibited by addition of an excess of nonlabeled TIR and not by poly (dI-dC) (Figure 1C). On the basis of a titration experiment, we calculated an approximate $K_{\mathrm{D}}$ value of $8.5 \times 10^{-8} \mathrm{M}$ for the MetnaseTIR interaction (Figure 1D).

A recent sequence specificity study identified $19 \mathrm{bp}$ of core Metnase binding sites within the TIR sequence (1). To deepen our understanding of the sequence specificity of Metnase DNA binding activity, we compared the TIR with four mutants, MARx11 (two-nucleotide mutation at the second half), MAR1M (five-nucleotide mutation at the first half), MAR2M (five-nucleotide mutation at the second half), and MAR3M (five-nucleotide mutation each at the first and second halves) (Figure 2A). Metnase was able to interact with MARx11 DNA, although the interaction was significantly weaker than the Metnase-TIR interaction (Figure 2B). On the other hand, consistent with previously reported results (1), Metnase (SETMAR) failed to interact with either MAR1M or MAR2M, suggesting that the TIR consists of two key regions within the TIR.

R432 within the HTH Motif Is Essential for the MetnaseTIR Interaction. The previous study showed that the helixturn-helix (HTH) motif (residues 415-435) within the transposase domain is sufficient for the Metnase-TIR interaction (1). In an effort to find the amino acid(s) that are critical for the TIR-specific DNA binding, we compared wtMetnase with two deletion mutants [dS-Metnase(329-671) lacking the SET domain dS-Metnase(433-671) lacking the SET and the HTH motif] and the Arg-to-Ala mutation at R432 (R432A) (Figure 3A,B). R432 was selected for a substitution mutation because, in Drosophila Mos-1 transposase, the arginine residue (R106) at the HTH motif is crucial for its interaction with TIR (12). dS-Metnase(329$671)$ retained its TIR binding activity, while dS-Metnase(433-671) did not (Figure 3C). Similar to Mos-1, R432 is critical for sequence-specific recognition, as the R432A 

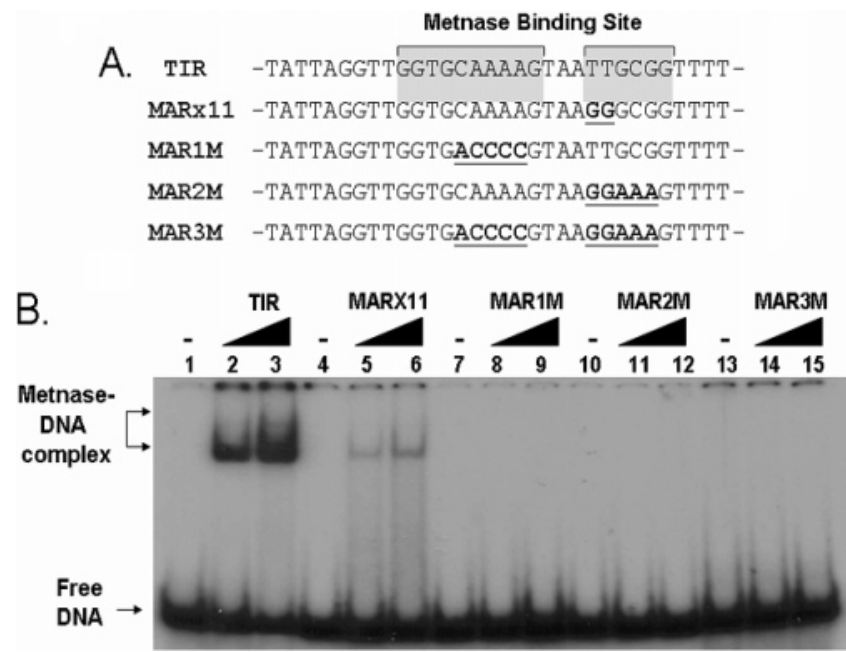

FIGURE 2: Sequence specificity of the Metnase-TIR interaction. (A) DNA sequences of TIR and four mutants. Mutation sites are indicated as underlined bold letters. (B) TIR-specific interaction of Metnase with DNA. Reaction mixtures contained $200 \mathrm{fmol}$ of ${ }^{32}$ P-labeled TIR (lanes 1-3), MARx11 (lanes 4-6), MAR1M (lanes 7-9), MAR2M (lanes 10-12), and MAR3M (lanes 13-15) in the presence of increasing amounts of wt-Metnase $(1.0 \mathrm{pmol}$ in lanes $2,5,8,11$, and 14 and 2.0 pmol in lanes $3,6,9,12$, and 15).

A.

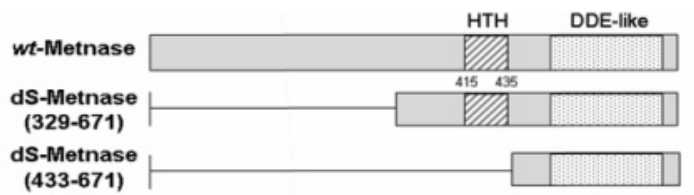

B.

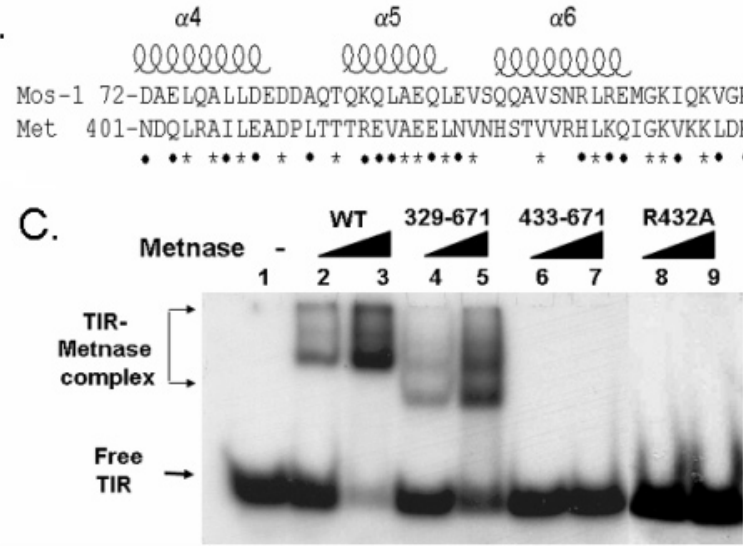

FIGURE 3: R432 within the HTH motif is essential for the MetnaseTIR interaction. (A) Schematic diagram of wt-Metnase and two deletion mutants, dS-Metnase(433-671) and dS-Metnase(329671). (B) Alignment of the HTH motif within the transposase domain of Metnase and a Drosophila transposase, Mos1. Identical and similar amino acids are marked with asterisks and circles, respectively. (C) In vitro DNA binding activity of wt-Metnase and the mutants. TIR-specific interaction of wt-Metnase, two deletion mutants [dS-Metnase(329-671) and dS-Metnase(433-671)], and the R432A. Reaction mixtures containing wt-Metnase (lanes 2 and 3), dS-Metnase(329-671) (lanes 4 and 5), dS-Metnase(433-671) (lanes 6 and 7), and R432A (lanes 8 and 9) were incubated with $200 \mathrm{fmol}$ of ${ }^{32} \mathrm{P}$-labeled TIR for their interaction with TIR DNA. Where indicated, either 1.0 pmol (lanes $2,4,6$, and 8) or 2.0 pmol (lanes 3, 5, 7, and 9) of purified protein was included.

mutation abolishes its TIR-specific DNA binding activity (Figure 3C).

Metnase Possesses a Unique DNA Nicking and Endonuclease Activity Different from Those of Other Eukaryotic Transposases. A recent study showed that the transposase domain of human Metnase (SETMAR) retained DNA nicking and strand transfer activity (11). Since Metnase (SETMAR) is a SET-transposase fusion protein that functions in DNA repair (2), it may have a unique DNA cleavage activity different from those of other eukaryotic transposases. We therefore examined the sequence specificity of Metnase's DNA cleavage activity. Fractions from the heparin-Sepharose column showed a single peak at $80 \mathrm{kDa}$ (Figure 4A) that was recognized by both anti-FLAG and anti-Metnase antibodies on immunoblot analysis (panels $\mathrm{B}$ and $\mathrm{C}$ of Figure 4, respectively). Fractions containing Metnase correlated with both single-strand nicking and double-strand endonuclease activities that converted supercoiled replicative form I (RFI) pBS DNA into nicked DNA (RFII) and linearized DNA (RFIII) (Figure 4D). Although Metnase (SETMAR) contains a putative cysteine-rich zinc-binding motif (preSET domain) that is often required for DNA binding or endonuclease activity in a redox-dependent manner $(13,14)$, its DNA cleavage activity was not affected by DTT (data not shown). Since Metnase possesses TIR-specific DNA binding activity (Figures 1 and 2) (1), we next examined whether DNA cleavage occurs at a specific sequence. If so, we would expect to see a unique cleavage pattern of the DNA template following Metnase exposure. Linearized duplex DNA (RFIII) was isolated from an agarose gel following incubation of Metnase with RFI DNA. This RFIII DNA was end-labeled with T4 PNK and then digested with a restriction enzyme (EcoRI or ScaI) that cuts only once. A number of cleavage products were identified following incubation of RFI DNA with Metnase (Figure 4E). Direct DNA sequence analysis of 5 cleavage products revealed that Metnase-mediated DNA cleavage occurred at no defined DNA sequence (Figure 4F).

The Transposase Domain but Not the HTH Motif Is Essential for Metnase's DNA Cleavage Activity. To further understand the relationship between DNA binding and DNA cleavage of Metnase (SETMAR), wt-Metnase and three deletion mutants [dT-Metnase(1-414), dS-Metnase(433671), and dS-Metnase(329-671)] (Figure 5A) were purified to near homogeneity (Figure 5B) and examined for DNA cleavage activity. Deletion of the transposase domain showed no detectable DNA nicking and/or endonuclease activity, while the two SET domain deletion mutants were able to convert RFI to RFII and RFIII by DNA cleavage (Figure 5C), suggesting that the transposase domain is responsible for Metnase's DNA cleavage activity. It is interesting to note that the dS-Metnase(433-671) lacking the HTH motif also exhibited DNA cleavage activity (Figure 5C, lanes 8 and 9), suggesting that the HTH motif is not essential for Metnase's DNA cleavage activity.

Metnase-Mediated DNA Cleavage Is Not Dependent on the TIR Sequence. Since the HTH motif essential for the Metnase-TIR interaction is not required for its DNA cleavage activity, we reasoned that Metnase-mediated DNA cleavage is independent of binding to the TIR sequence. To test this, increasing amounts of purified wt-Metnase (SETMAR) were incubated with ${ }^{32} \mathrm{P}$-labeled TIR, MARx11, or MAR3M, and the cleavage products were analyzed by denaturing $16 \%$ PAGE. A single cleavage product two nucleotides in size was mainly produced when Metnase was incubated with all three DNA substrates (Figure 6A). While this result is consistent with a recent observation that Metnase targets dinucleotide (11), it suggests that TIR sequence 

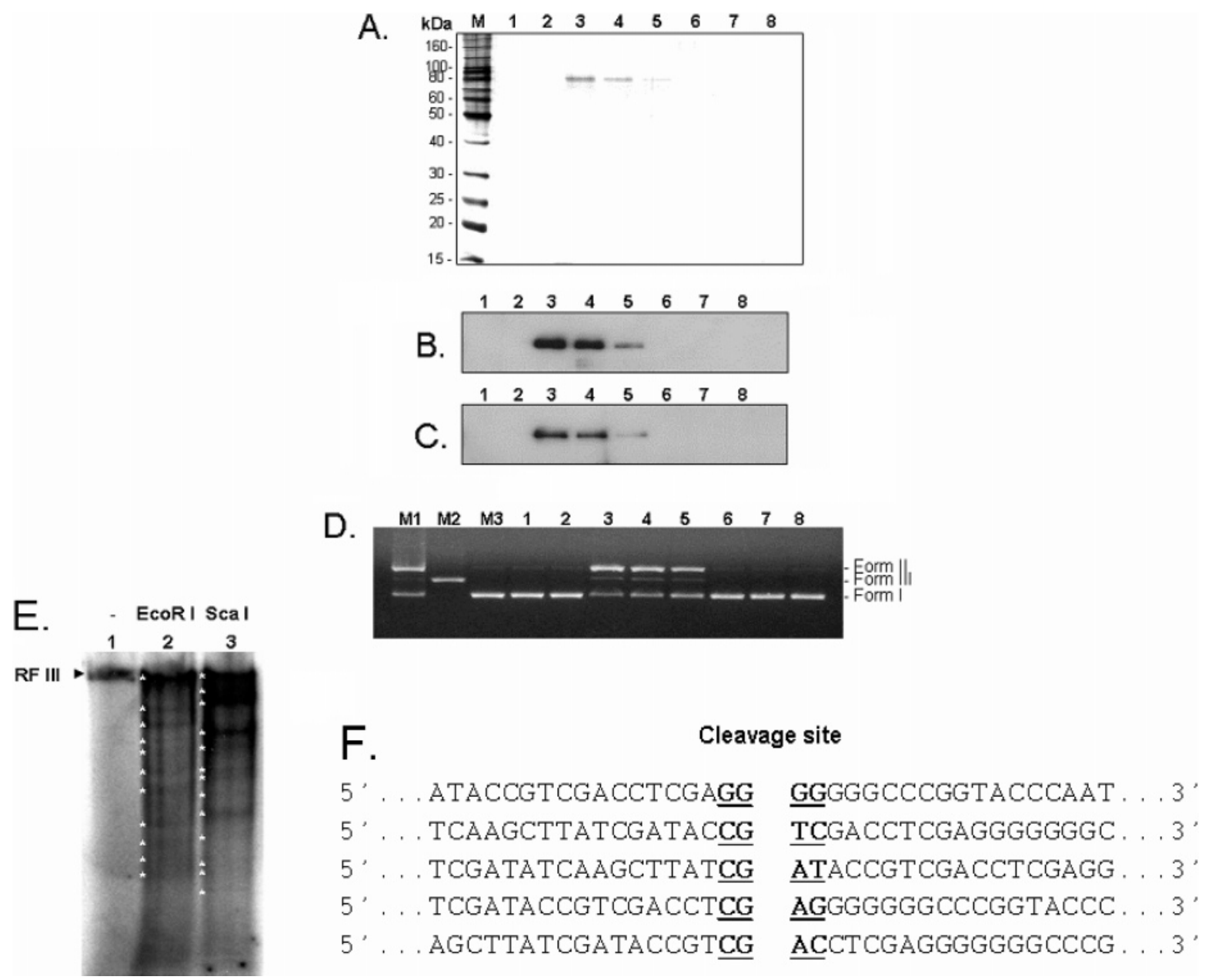

FIGURE 4: Metnase (SETMAR) possesses DNA nicking and endonuclease activity that targets no defined consensus sequence. (A) SDSPAGE (10\%) and silver staining of Metnase-containing fractions from heparin-Sepharose column chromatography. (B) Western blot analysis of Metnase-containing fractions shown in panel A using an anti-FLAG monoclonal antibody. (C) Western blot analysis of Metnase-containing fractions using an anti-Metnase polyclonal antibody. (D) DNA nicking and endonuclease activity of Metnase-containing fractions visualized on an agarose gel (1\%). Replicative form (RF) I, II, and III represent closed circular (supercoiled), open circular (nicked), and linearized pBS DNA, respectively. Lanes M1 (RFI + RFII), M2 (RFIII), and M3 (RFI) contained the marker DNAs. (E) Analysis of Metnasemediated DNA cleavage products. Following incubation of Metnase with RFI DNA (100 ng of pBS), the linearized DNA products (lane 1) were isolated from a $1 \%$ agarose gel and labeled with $\left[\gamma-{ }^{32} \mathrm{P}\right] \mathrm{ATP}$ and T4 PNK. This labeled RFIII DNA was incubated with either EcoRI (lane 2) or ScaI (lane 3) at $37^{\circ} \mathrm{C}$ for $60 \mathrm{~min}$. Following incubation, DNA samples were analyzed by $2.0 \%$ agarose gel electrophoresis and autoradiography. The cleavage products are marked with asterisks. (F) DNA sequence analysis of Metnase-mediated DNA cleavage products. To determine the sequence of the Metnase cleavage sites, the linearized DNA products (lane 1 in panel A) were treated with EcoRI. DNA fragments were cloned into pBluescript II SK+ (Stratagene), and the plasmid DNA containing inserts with fewer than 200 nucleotides were sequenced for cleavage site analysis.

essential for Metnase's DNA binding activity is not required for its DNA cleavage activity. In addition to two nucleotides, other cleavage products $(18,26$, and 29 nucleotides) were observed when Metnase was incubated with non-TIR DNA, MAR3M (Figure 6A, lanes 8 and 9). Although Metnase (SETMAR) preferentially targeted dinucleotides for its DNA cleavage action, TA sequence at the $5^{\prime}$-end was not required for DNA cleavage action as DNA sequences other than TA at the $5^{\prime}$-end were equally effective in generating dinucleotides by Metnase (Figure 6B). Together, these results along with the cleavage site analysis (Figure 4F) suggest that Metnase's DNA cleavage activity is not dependent on the TIR sequence.

To deepen our understanding of the relationship between Metnase's DNA binding and DNA cleavage activities, we carried out an equilibrium DNA binding assay using fluorescence polarization (FP). FP is a measure of the rate at which an object tumbles or rotates in three-dimensional space $(15,16)$. Binding of Metnase to a 5 -fluorescein-labeled DNA will slow its rotation and increases the FP value. A titration of Metnase showed an increase in the FP value with both TIR and MAR3M DNA in the presence of $50 \mathrm{mM} \mathrm{NaCl}$ (Figure 7A) but showed little or no FP values in reaction mixtures containing $150 \mathrm{mM} \mathrm{NaCl}$ (Figure 7B). This result suggests that, in contrast to a stable Metnase-DNA interaction, an equilibrium binding of Metnase to DNA is TIRindependent and correlates with its DNA cleavage activity.

D483 within the DDE-like Motif Is Essential for Metnase's DNA Cleavage Activity. Both prokaryotic and eukaryotic transposases have a conserved DDE motif within the catalytic domain that plays a crucial role in DNA cleavage action (17-22). Metnase (SETMAR) also possesses a DDE-like motif $(483 \mathrm{D}, 575 \mathrm{D}$, and $610 \mathrm{~N})$ within the transposase domain (Figure 8A). We therefore examined whether the DDE-like motif is involved in Metnase's DNA cleavage activity. We prepared a mutant protein that has the Asp-to- 
A.

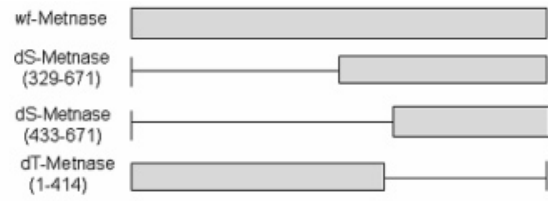

B.

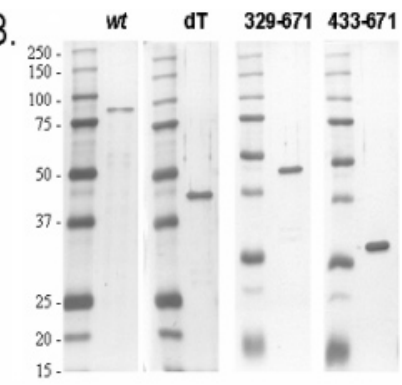

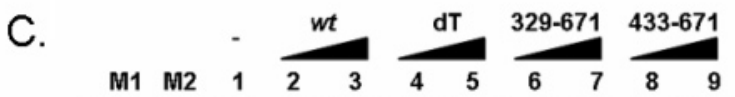

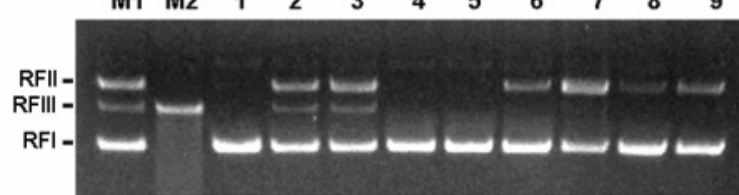

FIGURE 5: HTH motif that is not essential for Metnase-mediated DNA nicking and endonuclease activity. (A) wt-Metnase and three deletion mutants [dS-Metnase(433-671), dS-Metnase(329-671), and dT-Metnase(1-414)]. (B) SDS-PAGE (10\%, silver staining) of purified $w t$-Metnase and three deletion mutants used in this study. (C) Reaction mixtures containing 100 ng of RFI DNA were incubated with an increasing amount of either wt-Metnase or the mutants (0.7 pmol in lanes 2, 4, 6, and 8 and 1.4 pmol in lanes 3, 5, 7, and 9) and analyzed for DNA cleavage by $1 \%$ agarose gel electrophoresis.
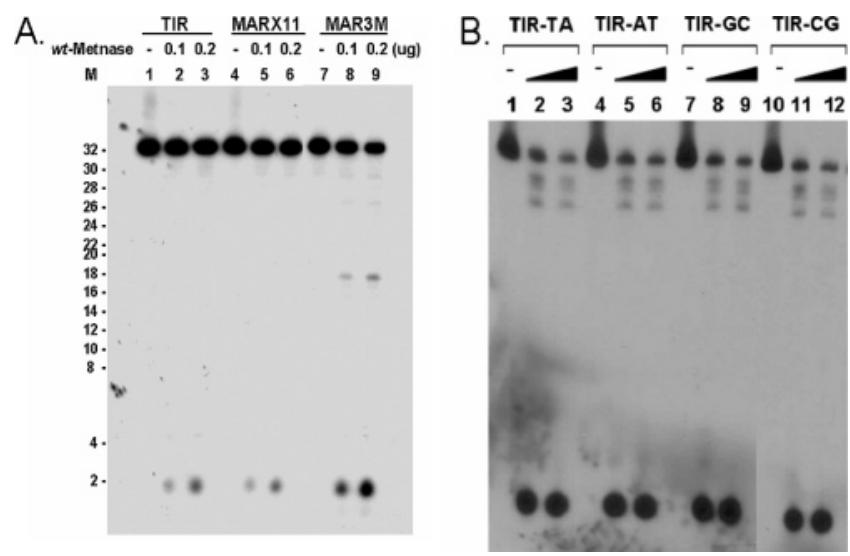

FIGURE 6: Metnase-mediated DNA cleavage occurs in a manner independent of TIR sequence. (A) Reaction mixtures $(20 \mu \mathrm{L})$ containing $20 \mathrm{fmol}$ of $5^{\prime}-{ }^{32} \mathrm{P}$-labeled TIR (lanes 1-3), MARx11 (lanes 4-6), or MAR3M (lanes 7-9) were incubated with 0 (lanes 1, 4, and 7), 0.1 (lanes 2, 5, and 8), and $0.2 \mu \mathrm{g}$ (lanes 3, 6, and 9) of wt-Metnase in the presence of $1 \mathrm{mM} \mathrm{MgCl} 2$. After incubation at $37{ }^{\circ} \mathrm{C}$ for $60 \mathrm{~min}$, reaction mixtures were analyzed by $16 \%$ denaturing PAGE for DNA cleavage. Lane M contained 5'-labeled size markers generated by the Maxam-Gilbert chemical cleavage ( $\mathrm{G}$ and $\mathrm{G}+\mathrm{A}$ reactions) (30). (B) Metnase-mediated DNA cleavage of the TIR DNA at a TA (lanes 1-3), AT (lanes 4-6), GC (lanes 7-9), or CG (lanes 10-12) dinucleotide at the 5'-end. Where indicated, 0 (lanes 1, 4, 7, and 10), 0.1 (lanes 2, 5, 8, and 11), and $0.2 \mu \mathrm{g}$ (lanes 3, 6, 9, and 12) of wt-Metnase was added.

Ala substitution at amino acid 483 (D483A) using the same purification protocol that was used for wt-Metnase (Figure 8B). Compared with wt-Metnase, D483A showed little or no DNA cleavage activity even when an excess of the protein was incubated with either TIR or MAR3M (Figure 8C). We also examined Metnase's DNA cleavage activity using a 50mer of duplex DNA that has a sequence completely different from that of TIR. Incubation of wt-Metnase with a 50-mer duplex DNA produced multiple cleavage products, while the D483A mutant showed no detectable DNA cleavage (data not shown). Together, these results suggest that Metnase (SETMAR) requires the DDE-like motif within the transposase domain for its DNA cleavage activity.
A. $(50 \mathrm{mM} \mathrm{NaCl})$
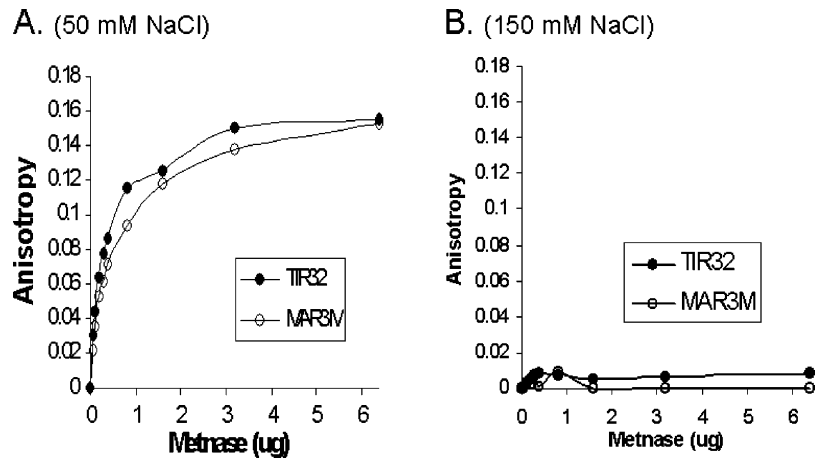

FIGURE 7: Equilibrium binding of Metnase to TIR and non-TIR (MAR3M) DNA. Using the fluorescence spectrophotometer (Varian), a change in fluorescence polarization (anisotropy) was measured in a $500 \mu \mathrm{L}$ assay format containing various amounts of Metnase and a fluorescein-labeled 32-mer of either TIR or MAR3M $(10 \mathrm{nM})$

\section{DISCUSSION}

Metnase (SETMAR) is a novel DNA repair protein that has a conserved functional transposase coding sequence spliced into a human gene encoding a SET domain protein (1-4). Recent studies showed that Metnase (SETMAR) preserves most of the activities of the ancestral Hsmar1 transposase, including TIR-specific DNA binding, assembly of a paired end complex, and cleavage of the $5^{\prime}$-end of the TIR element, and integration at a TA dinucleotide target site $(1-4)$. In this study, we found that Metnase (SETMAR) possesses a unique DNA nicking and/or endonuclease activity that is independent of its TIR-specific DNA binding. Given that DNA transposons are generally accepted as being extinct in humans (7), Metnase's DNA cleavage activity has evolved into a unique function(s) in processes other than DNA transposition such as DNA repair (2).

Metnase (SETMAR) recognizes the 19-mer core of the 5 '-terminal inverted repeats (TIR) of the Hsmar1 element (Figure 1) $(1,11)$. In Mos-1, R106 is critical for sequencespecific recognition, as the R106A mutation abolishes its binding activity (12). Similarly, R432 within the HTH motif 


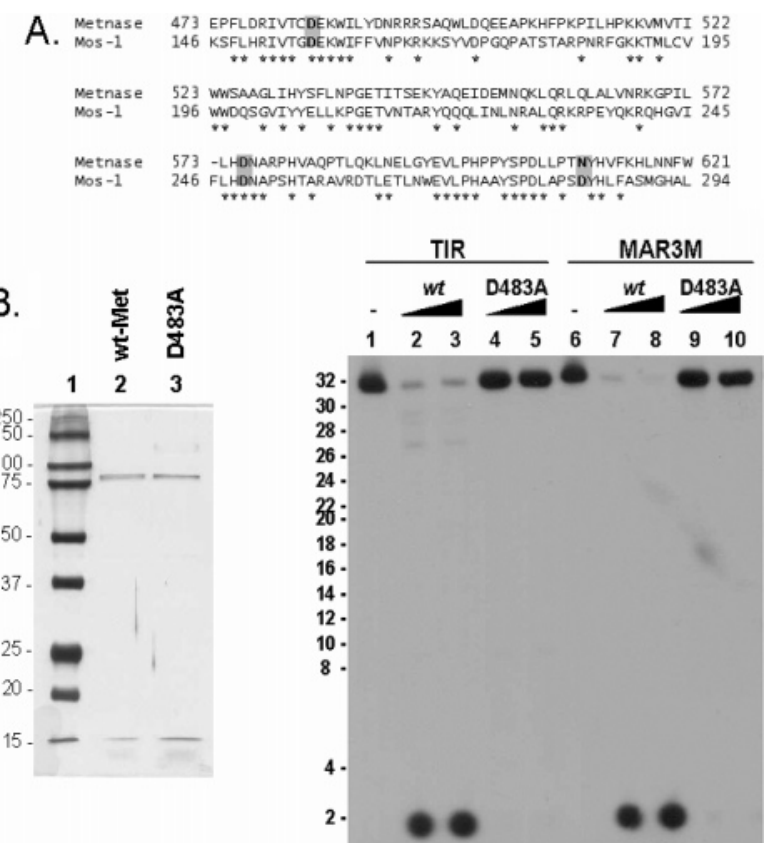

FIGURE 8: DDE-like motif within the transposase domain that is essential for Metnase's DNA cleavage activity. (A) Alignment of Metnase's DDE motif with Drosophila transposase, Mos-1. The DDE motif (residues 483, 575, and 610) is highlighted in gray. Conserved sequences are marked with asterisks. (B) SDS-PAGE (10\%, silver staining) of purified wt-Metnase and the mutant (D483A) used in this study. (C) Cleavage of TIR and MAR3M with wt-Metnase and the D483A mutant. Reaction mixtures (20 $\mu \mathrm{L}$ ) containing $20 \mathrm{fmol}$ of $5^{\prime}-{ }^{32} \mathrm{P}$-labeled DNA substrate were incubated with wt-Metnase $(0.4 \mu \mathrm{g}$ in lanes 2 and 7 and $0.8 \mu \mathrm{g}$ in lanes 3 and 8$)$ or the D483A mutant $(0.4 \mu \mathrm{g}$ in lanes 4 and 9 and $0.8 \mu \mathrm{g}$ in lanes 5 and 10). Cleavage products were analyzed by $16 \%$ denaturing PAGE for DNA cleavage. Lane M contained 5'labeled size markers.

is essential for Metnase's TIR-specific DNA binding activity (Figure 3C). In transposase action, TIR-specific interaction is tightly coupled to its DNA cleavage activity. In this study, however, we found that the TIR sequence was not required for Metnase's DNA cleavage activity. Other evidence also supports the possibility that Metnase's DNA cleavage action is not coupled to the Metnase-TIR interaction. First, the HTH motif is essential for both DNA binding and DNA cleavage activities in eukaryotic transposases (12), while it is required for only the TIR-specific binding and not for DNA cleavage action (Figures 3B and 5C). Uncoupling of TIRspecific DNA binding and DNA cleavage activities was also supported by an observation that Metnase-mediated DNA cleavage occurred at sites with no defined consensus sequence (Figure 4F). Together, these observations suggest that Metnase, instead of the typical cut and paste of transposase action $(12,23)$, possesses TIR-independent DNA cleavage activity that may be involved in other DNA metabolism such as DNA repair (2).

All eukaryotic transposases contain a DDE motif at the C-terminus that is responsible for DNA cleavage and joining reactions $(1,2,12,17,19,24)$. Metnase's transposase region contains the DDE-like motif (D483, D575, and N610) that is shared among the transposase family with polynucleotide phosphotransferase activity (25) and retroviral integrases (5, 26). A point mutation at D483 failed to support DNA cleavage activity (Figure 8), suggesting that the DDE-like motif functions as a catalytic core directly interacting with divalent cation, which is required for the DNA strand cleavage and joining reaction of retroviral integrase and eukaryotic transposases $(11,19,27-29)$. Unlike retroviral integrase or DNA transposase, however, the third D/E amino acid is mutated to $\mathrm{N}$ in human Metnase and the cognate SETMAR genes in primates $(1,2)$.

The functional implication of the Metnase-TIR interaction is obviously a key issue. Given that approximately half of the human genome is derived from transposable elements (7) and there are more than 7000 potential Metnase (SETMAR) binding sites in the human genome sequence $(1,4$, 7,11 ) (data not shown), the recruitment of the Metnase to the DNA binding sites may form a gene regulatory network (4). Potential Metnase binding sites (MBS) are also found in the coding region(s) of 45 proteins, including DNA repair factors such as FANCD2, Mre11, NBS1, Werner protein, BRCA2 interacting protein, etc. (data not shown). Although the specific role the Metnase-TIR interaction has in vivo is not clear, it may be tied to its two biochemical functions, histone lysine methyltransferase and DNA cleavage activity. A potential role for the Metnase-DNA interaction in DNA repair and genomic integration is obviously a major issue (2); however, a genetic regulatory network composed of Metnase's DNA binding domain (transposase domain) and its cognate binding sites dispersed throughout the genome may also provide a means of uncovering Metnase-mediated methylation of histone $\mathrm{H} 3$ at lysine 4 and/or lysine 36 to particular sites in the genome where it could affect gene expression or other biological processes.

\section{ACKNOWLEDGMENT}

We thank Drs. David Finnegan and Angela Dawson for valuable suggestions and Heather Ramsey for her technical assistance during the course of this work.

\section{REFERENCES}

1. Cordaux, R., Udit, S., Batzer, M. A., and Feschotte, C. (2006) Birth of a chimeric primate gene by capture of the transposase gene from a mobile element, Proc. Natl. Acad. Sci. U.S.A. 103, 8101-8106.

2. Lee, S. H., Oshige, M., Durant, S. T., Rasila, K. K., Williamson, E. A., Ramsey, H., Kwan, L., Nickoloff, J. A., and Hromas, R. (2005) The SET domain protein Metnase mediates foreign DNA integration and links integration to nonhomologous end-joining repair, Proc. Natl. Acad. Sci. U.S.A. 102, 18075-18080.

3. Robertson, H. M., and Zumpano, K. L. (1997) Molecular evolution of an ancient mariner transposon, Hsmar1, in the human genome, Gene 205, 203-217.

4. Jordan, I. K. (2006) Evolutionary tinkering with transposable elements, Proc. Natl. Acad. Sci. U.S.A. 103, 7941-7942.

5. Craigie, R. (2001) HIV integrase, a brief overview from chemistry to therapeutics, J. Biol. Chem. 276, 23213-23216.

6. Skalka, A. M., and Katz, R. A. (2005) Retroviral DNA integration and the DNA damage response, Cell Death Differ. 12 (Suppl. 1), 971-978.

7. Lander, E. S., Linton, L. M., Birren, B., Nusbaum, C., Zody, M. C., et al. (2001) Initial sequencing and analysis of the human genome, Nature 409, 860-921.

8. Agrawal, A., Eastman, Q. M., and Schatz, D. G. (1998) Transposition mediated by RAG1 and RAG2 and its implications for the evolution of the immune system, Nature 394, 744-751.

9. Bundock, P., and Hooykaas, P. (2005) An Arabidopsis hAT-like transposase is essential for plant development, Nature 436, 282284.

10. Ivics, Z., Kaufman, C. D., Zayed, H., Miskey, C., Walisko, O., and Izsvak, Z. (2004) The Sleeping Beauty transposable element: Evolution, regulation and genetic applications, Curr. Issues Mol. Biol. 6, 43-55. 
11. Liu, D., Bischerour, J., Siddique, A., Buisine, N., Bigot, Y., and Chalmers, R. (2007) The human SETMAR protein preserves most of the activities of the ancestral Hsmar1 transposase, Mol. Cell. Biol. 27, 1125-1132.

12. Zhang, L., Dawson, A., and Finnegan, D. J. (2001) DNA-binding activity and subunit interaction of the mariner transposase, Nucleic Acids Res. 29, 3566-3575.

13. Arai, K., Morishita, K., Shinmura, K., Kohno, T., Kim, S. R., Nohmi, T., Taniwaki, M., Ohwada, S., and Yokota, J. (1997) Cloning of a human homolog of the yeast OGG1 gene that is involved in the repair of oxidative DNA damage, Oncogene 14, 2857-2861.

14. Park, J. S., Wang, M., Park, S. J., and Lee, S. H. (1999) Zinc finger of replication protein $\mathrm{A}$, a non-DNA binding element, regulates its DNA binding activity through redox, J. Biol. Chem. 274, 29075-29080.

15. Lundblad, J. R., Laurance, M., and Goodman, R. H. (1996) Fluorescence polarization analysis of protein-DNA and proteinprotein interactions, Mol. Endocrinol. 10, 607-612.

16. Pavski, V., and Le, X. C. (2003) Ultrasensitive protein-DNA binding assays, Curr. Opin. Biotechnol. 14, 65-73.

17. Swanson, P. C. (2001) The DDE motif in RAG-1 is contributed in trans to a single active site that catalyzes the nicking and transesterification steps of V(D)J recombination, Mol. Cell. Biol. 21, 449-458.

18. Junop, M. S., and Haniford, D. B. (1997) Factors responsible for target site selection in Tn10 transposition: A role for the DDE motif in target DNA capture, EMBO J. 16, 2646-2655.

19. Neiditch, M. B., Lee, G. S., Landree, M. A., and Roth, D. B. (2001) RAG transposase can capture and commit to target DNA before or after donor cleavage, Mol. Cell. Biol. 21, 4302-4310.

20. Tavakoli, N. P., DeVost, J., and Derbyshire, K. M. (1997) Defining functional regions of the IS903 transposase, J. Mol. Biol. 274 , 491-504.

21. Davies, D. R., Mahnke Braam, L., Reznikoff, W. S., and Rayment, I. (1999) The three-dimensional structure of a Tn5 transposase- related protein determined to $2.9-\AA$ resolution, J. Biol. Chem. 274 , 11904-11913.

22. Kim, D. R., Dai, Y., Mundy, C. L., Yang, W., and Oettinger, M. A. (1999) Mutations of acidic residues in RAG1 define the active site of the V(D)J recombinase, Genes Dev. 13, 3070-3080.

23. Lampe, D. J., Churchill, M. E., and Robertson, H. M. (1996) A purified mariner transposase is sufficient to mediate transposition in vitro, EMBO J. 15, 5470-5479.

24. Dawson, A., and Finnegan, D. J. (2003) Excision of the Drosophila mariner transposon Mos1. Comparison with bacterial transposition and V(D)J recombination, Mol. Cell 11, 225-235.

25. Ton-Hoang, B., Turlan, C., and Chandler, M. (2004) Functional domains of the IS1 transposase: Analysis in vivo and in vitro, Mol. Microbiol. 53, 1529-1543.

26. Chiu, T. K., and Davies, D. R. (2004) Structure and function of HIV-1 integrase, Curr. Top. Med. Chem. 4, 965-977.

27. Allingham, J. S., Pribil, P. A., and Haniford, D. B. (1999) All three residues of the Tn 10 transposase DDE catalytic triad function in divalent metal ion binding, J. Mol. Biol. 289, 11951206.

28. Daniel, R., Greger, J. G., Katz, R. A., Taganov, K. D., Wu, X. Kappes, J. C., and Skalka, A. M. (2004) Evidence that stable retroviral transduction and cell survival following DNA integration depend on components of the nonhomologous end joining repair pathway, J. Virol. 78, 8573-8581.

29. Maignan, S., Guilloteau, J. P., Zhou-Liu, Q., Clement-Mella, C., and Mikol, V. (1998) Crystal structures of the catalytic domain of HIV-1 integrase free and complexed with its metal cofactor: High level of similarity of the active site with other viral integrases, J. Mol. Biol. 282, 359-368.

30. Maxam, A. M., and Gilbert, W. (1977) A new method for sequencing DNA, Proc. Natl. Acad. Sci. U.S.A. 74, 560-564.

BI7005477 